\title{
Equivalence between local Fermi gas and shell models in inclusive muon capture from nuclei
}

\author{
J.E. Amaro ${ }^{1, a}$, C. Maieron ${ }^{2}$, J. Nieves ${ }^{1}$, and M. Valverde ${ }^{1}$ \\ 1 Departamento de Física Moderna, Universidad de Granada, Granada 18071, Spain \\ 2 INFN, Sezione di Catania, Via Santa Sofia 64, 95123 Catania, Italy
}

Original article: Eur. Phys. J. A 24, 343-353 (2005) DOI: 10.1140/epja/i2005-10034-2

Received: 25 November 2005 /

Published online: 16 December 2005 - C) Società Italiana di Fisica / Springer-Verlag 2005

An input error in the sign of the muon binding energy was made in the calculations for ${ }^{16} \mathrm{O},{ }^{40} \mathrm{Ca}$ and ${ }^{208} \mathrm{~Pb}$ in our paper. Hence an incorrect energy balance affects table 4 and figs. 4, 6 and 8 published therein. This error is corrected in the table and in the figures presented on the following pages.

Although the numerical results for total capture rates change, the relative differences between LFG and shell model are not much affected. The main trends remain the same and so do our conclusions.

It should also be noted another error in table 2 in the $r_{L S}$ entry of WS2 parametrization for ${ }^{208} \mathrm{~Pb}$, which should read 1.21, consistently with WS1.

\footnotetext{
a e-mail: amaro@ugr.es
} 
Table 4. Integrated width in units of $10^{5} \mathrm{~s}^{-1}$ for the different nuclei and Woods-Saxon potentials, compared with the LFG results using the corresponding charge densities. The discrete contribution of the shell model is shown in the first column.

\begin{tabular}{lccccr}
\hline & & Discrete & Total & LFG & $\%$ \\
\hline \hline${ }^{12} \mathrm{C}$ & WS1 & 0.3115 & 0.4406 & 0.4548 & 3.2 \\
& WS2 & 0.3179 & 0.4289 & 0.4360 & 1.7 \\
& WS3 & 0.2746 & 0.5510 & 0.4732 & -14.1 \\
\hline${ }^{16} \mathrm{O}$ & WS1 & 1.124 & 1.267 & 1.346 & 6.2 \\
& WS2 & 0.584 & 1.107 & 1.378 & 24.4 \\
& WS3 & 1.143 & 1.316 & 1.373 & 4.3 \\
\hline${ }^{40} \mathrm{Ca}$ & WS1 & 27.72 & 34.87 & 34.81 & -0.1 \\
& WS2 & 26.34 & 31.70 & 33.07 & 4.3 \\
& WS3 & 24.91 & 30.64 & 33.19 & 8.3 \\
\hline${ }^{208} \mathrm{~Pb}$ & WS1 & 128.5 & 191.0 & 187.27 & -1.9 \\
& WS2 & 159.6 & 243.4 & 213.64 & -12.2 \\
\hline \hline
\end{tabular}

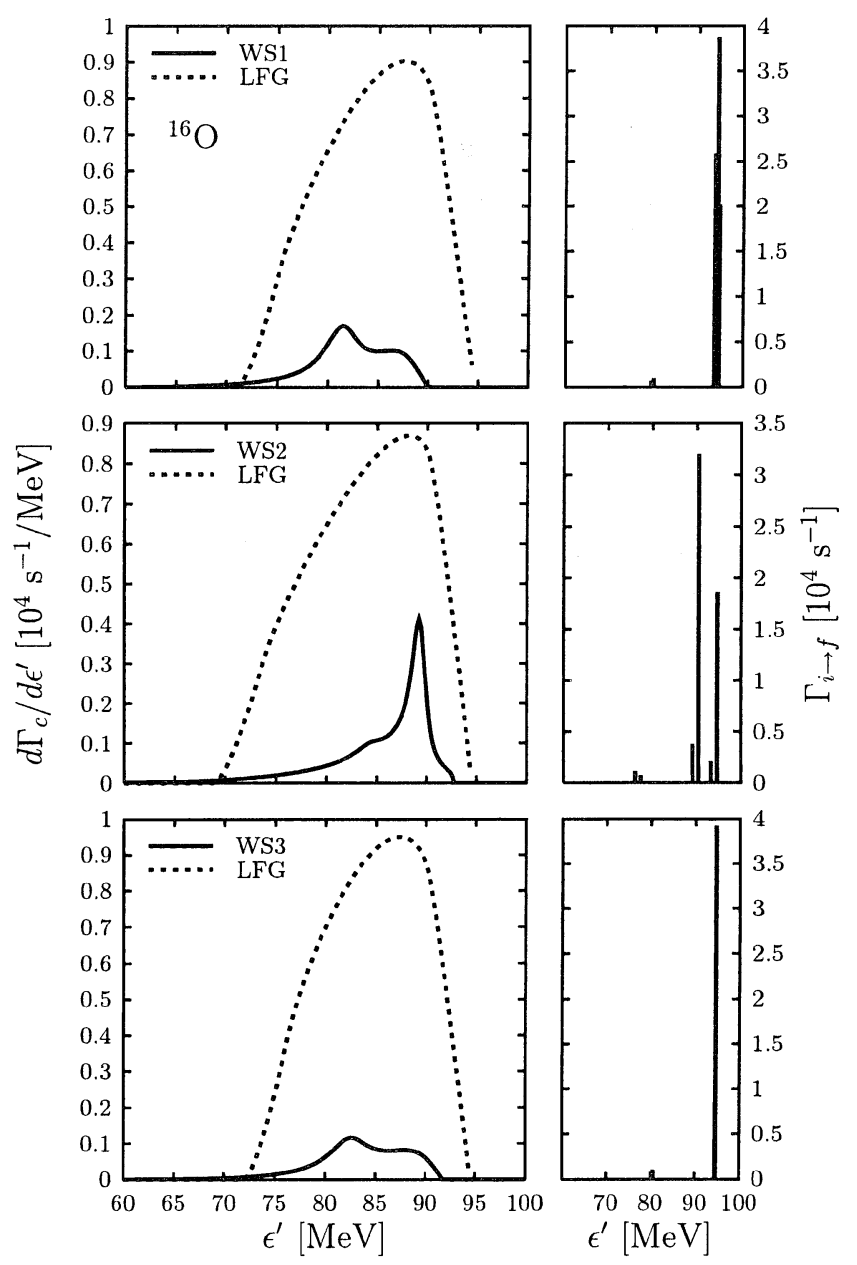

Fig. 4. Differential SM width of ${ }^{16} \mathrm{O}$ to the continuum (left panels) compared to the LFG, and partial widths to the discrete states (right panels), as a function of the neutrino energy, for the different WS potentials considered. 


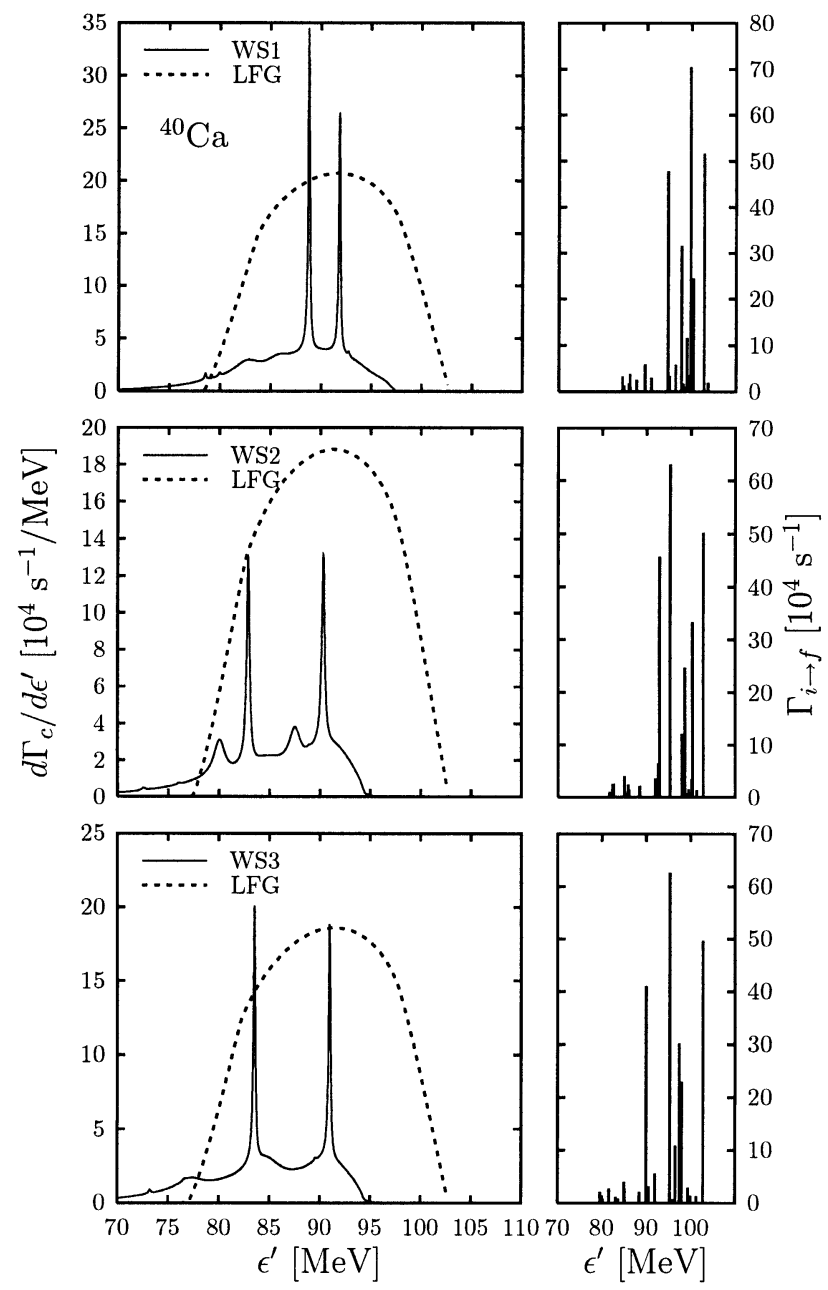

Fig. 6. The same as fig. 4 for ${ }^{40} \mathrm{Ca}$.
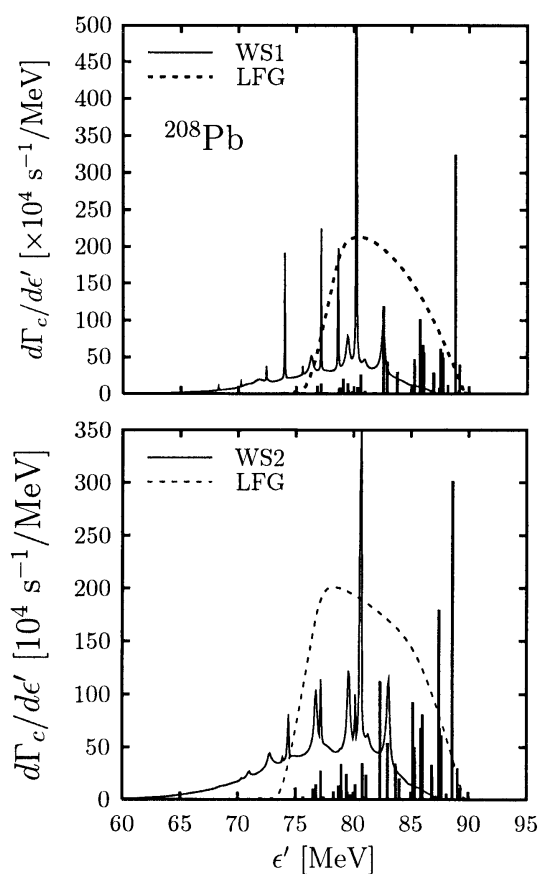

Fig. 8. The same as fig. 4 for ${ }^{208} \mathrm{~Pb}$. In this case we show the discrete contributions in units of $10^{4} \mathrm{~s}^{-1}$ in the same panel as the continuum one. 